\title{
Tongluo Xingnao effervescent tablet reverses memory deficit and reduces plaque load in APPswe/PS1dE9 mice
}

\author{
WENJUN FU ${ }^{1,2^{*}}$, YUAN DAI $^{3^{*}}$, TAO MA ${ }^{4 *}$, JIANGPING WEI ${ }^{1,2}$, HUAN CHEN $^{1,2}$ and SHIJUN XU ${ }^{1,2}$ \\ ${ }^{1}$ Institute of Meterial Medica Integration and Transformation for Brain Disorders, Chengdu University of \\ Traditional Chinese Medicine; ${ }^{2}$ College of Pharmacy, Chengdu University of Traditional Chinese Medicine, \\ Chengdu, Sichuan 610075; ${ }^{3}$ School of Health and Rehabilitation, Chengdu University of \\ Traditional Chinese Medicine; ${ }^{4}$ Experimental Center of Dongfang Hospital, \\ Beijing University of Chinese Medicine, Beijing 100078, P.R. China
}

Received December 30, 2016; Accepted November 29, 2017

DOI: $10.3892 /$ etm.2018.5897

\begin{abstract}
Alzheimer's disease (AD) is the most common type of dementia. Amyloid- $\beta$ (A $\beta$ )-induced neurodegeneration is hypothesized to be the primary pathological mechanism of AD. Tongluo Xingnao effervescent tablets (TXET), based on the traditional Chinese formula Qionggui Tang, have been used to treat AD and other types of dementia in China for decades. In the present study, the effects of TXET on cognition deficit, amyloid- $\beta$ production, amyloid precursor protein procession and $\beta$-secretase expression were investigated in the APPswe/PS1dE9 mouse model of AD. As expected, APPswe/PS1dE9 mice exhibited cognitive decline and higher levels of $A \beta$ and plaques in the brain compared with normal mice; however, these changes were attenuated following TXET treatment. Levels of C-terminal fragment (CTF)- $\beta$ protein were decreased following treatment with TXET; however, CTF- $\alpha$ levels were unaffected. Furthermore, TXET treatment did not decrease $\gamma$-secretase activity or levels of presenilin-1 (PS1), neprilysin or insulin-degrading enzyme. These results indicate that TXET may regulate $A \beta$ metabolism by downregulating the expression of $\beta$-secretase. The results of the present study have laid the foundation for the development of a Chinese medicinal compound with a $\beta$-secretase inhibitor as the target for the treatment of AD.
\end{abstract}

Correspondence to: Dr Shijun Xu, College of Pharmacy, Chengdu University of Traditional Chinese Medicine, 1166 LiuTai Road, Chengdu, Sichuan 610075, P.R. China

E-mail: docxu@126.com

*Contributed equally

Key words: Alzheimer's disease, Tongluo Xingnao effervescent tablet, APPswe/PS1dE9 mice, amyloid- $\beta$, amyloid precursor protein, C-terminal fragment $\beta$

\section{Introduction}

Alzheimer's disease (AD) is a neurodegenerative disease clinically characterized by cognitive and intellectual dysfunction; specific pathological hallmarks include the presence of senile plaques, cerebral amyloid angiopathy and neurofibrillary tangles (1). The number of people living with dementia is currently estimated to be $\sim 24$ million and its prevalence is forecast to double every 20 years until 2040 (2). The pathogenesis of AD is complex and has not yet been elucidated, however the results of numerous studies support the amyloid $\beta$ protein $(A \beta)$ hypothesis of AD development (3-5).

Amyloid- $\beta$ (A $\beta)$ is hypothesized to trigger the onset and development of AD. It also promotes oxidative stress, neurotropic signaling, Tau phosphorylation and synaptic dysfunction and activates inflammatory factors (6). The generation of $A \beta$ is dependent on the processing of amyloid precursor protein (APP), which involves two signaling pathways via sequential limited proteolysis. In the amyloidogenic pathway, APP is cleaved by $\beta$-secretase, generating a C-terminal fragment (CTF)- $\beta$ and soluble (s)APP- $\beta$ (7). CTF- $\beta$ is further cleaved by $\gamma$-secretase to generate $A \beta(8)$. Alternatively, APP may be processed via the nonamyloidogenic pathway, which is regulated by $\alpha$ - and $\gamma$-secretase. The cleavage of $\alpha$-secretase generates CTF- $\alpha$ and SAPP- $\alpha$, and CTF- $\alpha$ is subsequently cleaved by the $\gamma$-secretase complex to generate an APP intracellular domain and p3 peptides, thus impeding $A \beta$ production (7). It has been demonstrated that the cleavage pathway of APP serves an important role in the formation and accumulation of $A \beta(9)$.

A $\beta$ levels depend on APP processing, as well as on its elimination via transport processes, cell-mediated clearance and $A \beta$ degradation (10). It has been demonstrated that $A \beta$-degrading enzymes are required to degrade $A \beta$ (11). One such enzyme, neprilysin (NEP), degrades monomeric $A \beta$ and its oligomeric forms $A \beta_{40}$ and $A \beta_{42}$ (12). Furthermore, insulin-degrading enzyme (IDE) may be involved in $A \beta$ degradation, as indicated by Kurochkin and Goto (13). Therefore, NEP and IDE are the most promising $\mathrm{A} \beta$-degrading candidates.

Previous studies have focused on ways to decrease $A \beta$ production and reduce $\mathrm{A} \beta$ levels, which are the prime drug 
targets for $\mathrm{AD}(14,15)$. Therefore, targeting $\mathrm{A} \beta$ metabolism may be a potential method of treating AD. Currently, the development of novel drugs for AD treatment has focused on ways of decreasing $A \beta$ levels; however the results of clinical trials have not been promising $(16,17)$. Western medicine is a single-target, single-component and symptomatic treatment paradigm, while traditional Chinese medicine (TCM) therapy is a multi-target, multi-component and treatment strategy that has been applied to treat various diseases. Tongluo Xingnao effervescent tablets (TXET) are derived from Qionggui Tang [Ligusticum striatum DC. and Angelica sinensis (Oliv.) Diels], which has been used in Japan, China, South Korea and other East Asian countries for >2,000 years, usually in combination with Scutellaria baicalensis Georgi. In TCM, it is hypothesized that Qionggui Tang may be used to treat patients with various conditions. The addition of Scutellaria baicalensis Georgi may enhance the effect of Qionggui Tang on the efflux of pathogenic toxins. Therefore, the function of TXET involves promoting blood circulation to remove toxins, as well as improving memory and cognition (18-21). In a previous study by our group, TXET improved cognition and synaptic function; it also improved cerebral metabolism and mitochondrial function (18). Additionally, it was demonstrated that TXET may downregulate levels of cyclin-dependent kinase (CDK)5 and glycogen synthase kinase (GSK)-3, key proteins that regulate tau protein phosphorylation, thus inhibiting tau phosphorylation in an animal model of AD (19). Furthermore, TXET may increase acetylcholine (ACh) and choline acetyltransferase (ChAT) levels, thus enhancing the central cholinergic neuronal system in the hippocampi of rats with AD (20). Another study by our group demonstrated that TXET increases the expression of IDE, indicating that TXET may regulate the metabolism of $\mathrm{A} \beta$ to treat AD (21). In the present study, the anti-AD effect of TXET on the production and elimination of $\mathrm{A} \beta$ was investigated in AD transgenic mice.

\section{Materials and methods}

Reagents. TXET, based on the traditional Chinese formula Qionggui Tang [Ligusticum striatum DC and Angelica sinensis (Oliv.) Diels] plus Scutellaria baicalensis Georgi, is a patented prescription (Patent no. ZL 201310132096.2) (22) owned by the Affiliated Hospital of Chengdu University of Traditional Chinese Medicine (Chengdu, China) and used in the long-term treatment of dementia. Ligusticum striatum DC. (1,250 g) and Angelica sinensis (Oliv.) Diels (250 g) were extracted via supercritical carbon dioxide fluid extraction to collect residues and volatile oils. Ethanol was added to dissolve the volatile oils and the preparation was mixed with $\beta$-cyclodextrin dissolved in purified water $(1: 10)$ at $60^{\circ} \mathrm{C}$. The preparation contained these inclusion complexes and when the preparation was fully mixed, it was dried and the residue was crushed into a fine powder. Scutellaria baicalensis Georgi (750 g) and residues were extracted with $60 \%$ ethanol by heating under reflux 3 times, followed by filtration. The filtrate was concentrated to extractum such that the relative density was between 1.1 and 1.2; the extractum was dried using a vacuum dryer and made into grain. The inclusion complexes and the grain were blended with corresponding excipients and compressed into tablets, with each tablet weighing $1.2 \mathrm{~g}$ (active ingredient, $0.6 \mathrm{~g}$ ). TXET was provided by the Affiliated Hospital of Chengdu University of Traditional Chinese Medicine (Chengdu, China) and its quality analysis was performed by liquid chromatography (LC) assays, as previously described (23). The LC assay was performed using an LC-30A (Shimadzu Corporation, Kyoto, Japan) liquid chromatography system, with a LabSolutions 5.41.20 chromatography workstation (Shimadzu Corporation) and an Agilent Poroshell 120 EC-C 18 column (2.1x100 mm, $2.7 \mu \mathrm{m}$; Agilent Technologies Inc., Santa Clara, CA, USA). The results demonstrated that peaks of ferulic acid (18.5 min), baicalin (25.2 $\mathrm{min})$, baicalein (33.4 $\mathrm{min})$ and wogonin (48.9 min) appeared successively (data not shown), which indicated that the quality of TXET was high.

Aricept (cat. no. 100526A) was purchased from Eisai China Inc. (Eisai Co., Ltd., Tokyo, Japan) Human A $\beta 1-40$ (cat. no. KHB3481) and A $\beta 1-42$ ELISA kits (cat. no. KHB3441) were purchased from Invitrogen (Thermo Fisher Scientific, Inc., Waltham, MA, USA). A $\beta$ monoclonal antibodies (cat. no. 803015) were purchased from BioLegend, Inc. (San Diego, CA, USA). The DAB Kit for Immunohistochemistry was purchased from ZG ZSGB-Bio, Inc. (Beijing, China). APP (cat. no. 11090), CTF- $\alpha$ (cat. no. 11088) and CTF- $\beta$ (C99; cat. no. 10321) antibodies were purchased from Immuno-Biological Laboratories Co., Ltd, (Gunma, Japan). IDE (cat. no. ab32216) and NEP (cat. no. ab81688) antibodies were purchased from Abcam (Cambridge, UK). The presenilin-1 (PS1) antibody (cat. no. 5643) was purchased from Cell Signaling Technology, Inc. (Danvers, MA, USA). The $\beta$-actin antibody (cat. no. sc-47778), goat anti-rabbit IgG-HRP (cat. no. sc-2004) and goat anti-mouse IgG-HRP (cat. no. sc-2005) were provided by Santa Cruz Biotechnology, Inc. (Dallas, TX, USA). The $\gamma$-secretase activity kit (cat. no. FP003) was purchased from R\&D Systems, Inc. (Minneapolis, MN, USA) The BCA protein assay kit and radioimmunoprecipitation assay (RIPA) lysis buffer were purchased from Applygen Technologies, Inc. (Beijing, China).

Animals and administration procedure. A total of 60 3-month-old male APPswe/PS1dE9 mice and 15 age-matched C57BL/6J mice (20 $\pm 2 \mathrm{~g})$ were purchased from Beijing HFK Bioscience Co., Ltd. (Beijing, China; Certificate SCXK Jing 2009-0004;). In the animal observation room with a $12 \mathrm{~h}$ light/dark cycle, animals had ad libitum access to food and water and were housed under a suitable temperature $\left(22 \pm 2^{\circ} \mathrm{C}\right)$ and humidity $(65 \pm 5 \%)$. The treatment and care of animals adhered to the international laws and regulations indicated in the National Institutes of Health Guide for the Care and Use of Laboratory Animals (24). All animal protocols were approved by the Institutional Animal Care and Use Committee of Dongfang Hospital affiliated with the Beijing University of Chinese Medicine (Beijing, China).

The APPswe/PS1dE9 mice were randomly divided into four groups (each $\mathrm{n}=5)$ : A model group, an Aricept $(1 \mathrm{mg} / \mathrm{kg}$ ) group (positive control), a TXET low-dose $(0.45 \mathrm{~g} / \mathrm{kg})$ group and a TXET high-dose $(1.8 \mathrm{~g} / \mathrm{kg})$ group. C57BL/6J mice served as the normal control group, which alongside the model group was treated with an equal volume of sterile saline. A total of $10 \mathrm{ml} / \mathrm{kg} /$ day solution was administered to all mice intragastrically for 180 days. 
Morris water maze (MWM) test. All mice underwent the MWM test, which evaluates spatial learning and memory performance, on day 176 of administration. For the place navigation task, mice trained for 5 days consecutively, while the escape latency and total swimming distance were recorded in one $120 \mathrm{sec}$ session. The platform was then removed and the probe trial was conducted to record the distance each mouse crossed in the target area (where the platform was placed formerly) and the opposite area. All tests were performed at night.

Brain tissue preparation. For immunochemistry, three mice in each group were selected randomly and sacrificed under ether anesthesia by decapitation. Brains were harvested and fixed with $4 \%$ paraformaldehyde at $4^{\circ} \mathrm{C}$ overnight using perfusion fixation, followed by paraffin-embedding. Other mice $(34 \pm 4 \mathrm{~g})$ were sacrificed under ether anesthesia by decapitation; the brain tissue was immediately dissected, snap-frozen in liquid nitrogen and stored at $-80^{\circ} \mathrm{C}$ until processing.

Immunohistochemistry. Paraffin-embedded tissue was serially cut into 4 - $\mu$ m-thick sections. Sections were rinsed with $0.01 \mathrm{~mol} / 1 \mathrm{PBS}$ following dewaxing in xylene, rehydrated through decreasing concentrations of ethanol and washed in $0.01 \mathrm{~mol} / 1 \mathrm{PBS}$. For $\mathrm{A} \beta$-containing plaque immunostaining, antigens were unmasked by microwaving sections in $10 \mathrm{mmol} / \mathrm{l}$ citrate buffer, $(\mathrm{pH} 6.0)$ for $15 \mathrm{~min}$ and allowed to cool. Sections were then blocked with $5 \%$ goat serum (cat. no. ZLI-9022; OriGene Technologies, Inc., Beijing, China) for $1 \mathrm{~h}$ at room temperature, sections were treated with $3 \%$ $\mathrm{H}_{2} \mathrm{O}_{2}$ for 10 min to eliminate endogenous peroxidase activity. Following rinsing in PBS, sections were incubated with anti-A $\beta$ monoclonal antibodies $(1: 200)$ overnight at $4^{\circ} \mathrm{C}$. Following rinsing with PBS and the addition of Polymer Helper (polink-2 plus polymer HRP detection system; OriGene Technologies, Inc.; cat. no. PV-9001) incubated at $37^{\circ} \mathrm{C}$ for $\left.10 \mathrm{~min}\right)$, the sections were incubated with horseradish peroxidase conjugated Goat anti-Mouse IgG-Fragment crystallizable (1:500, cat. no. ab20043; Abcam) at $37^{\circ} \mathrm{C}$ for $1 \mathrm{~h}$ and stained with 3,3-diaminobenzidine of DAB kit (cat. no. ZLI-9018; OriGene Technologies, Inc.) for $6 \mathrm{~min}$ and hematoxylin for $10 \mathrm{~min}$ at room temperature. Following dehydration, sections were covered with neutral balsam. Using an inverted fluorescence microscope (IX71; Olympus Corporation, Tokyo, Japan; magnification $\mathrm{x} 200$ ), images of all sections of each group were captured and the number of $\mathrm{A} \beta$ plaques in the hippocampi were determined using Image-Pro Plus software (version 6.0; Media Cybernetics, Inc., Rockville, MD, USA).

ELISA. Human $\mathrm{A} \beta_{1-40}$ and $\mathrm{A} \beta_{1-42}$ ELISA kits were used to detect the concentration of $A \beta$ in the mouse brain. The brain tissue was homogenized and centrifuged at $5,000 \mathrm{x}$ g at $4^{\circ} \mathrm{C}$ for $15 \mathrm{~min}$ and the supernatant was used to detect $A \beta$ according to the manufacturer's protocol of the kits.

Western blot analysis. The expression of APP, CTF- $\alpha$, CTF- $\beta$, PS1, NEP and IDE proteins were measured by western blot analysis. Brain tissues were homogenized in RIPA lysis buffer and the homogenate was centrifuged at $4^{\circ} \mathrm{C}$ and $15,000 \mathrm{xg}$ for $20 \mathrm{~min}$. The protein concentration in the supernatant was determined using the BCA kit, following the manufacturer's protocol. A total of $40 \mu \mathrm{g}$ protein was separated using $10 \%$ SDS-PAGE and transferred to a polyvinylidene difluoride membrane (EMD Millipore, Billerica, MA, USA). The membrane was blocked using 5\% skimmed milk (cat. no. 1172GR100; BioFroxx GmbH, Einhausen, Germany) in TBS-T at room temperature for $1 \mathrm{~h}$ and incubated with primary antibodies (APP, PS1, NEP and IDE were diluted by $1: 1,000 ;$ CTF- $\alpha$ and CTF- $\beta$ were diluted by $1: 500)$ at $4^{\circ} \mathrm{C}$ overnight, and $\beta$-actin $(1: 1,000)$ was used as loading control. The membrane was then incubated with goat anti-mouse horseradish peroxidase conjugated (HRP) $\operatorname{IgG}(1: 2,000)$ and goat anti-rabbit IgG-HRP $(1: 5,000)$ at room temperature for $2 \mathrm{~h}$ and visualized using Super ECL Plus enhanced chemiluminescent kit (cat. no. P1010; Applygen Technologies Inc.). Membranes were then exposed to the GeneGnomeXRQ bio imaging system (Syngene, Frederick, MD, USA) and the images were quantified using Image J software version 1.46r (National Institutes of Health, Bethesda, MD, USA).

Secretase activity. The $\gamma$-secretase activity kit was used to detect the activity of $\gamma$-secretase in mouse brain tissues. The brain tissue was weighed, homogenized in a RIPA buffer and then centrifuged at $15,000 \mathrm{x} \mathrm{g}$ and $4^{\circ} \mathrm{C}$ for $20 \mathrm{~min}$. The supernatant was assessed following the manufacturer's protocol.

Statistical analysis. All data were analyzed by SPSS software (version 13.0; SPSS, Inc., Chicago, IL, USA) and the results are presented as the mean \pm standard deviation. For comparisons among groups, one-way analysis of variance (ANOVA) was used. $\mathrm{P}<0.05$ indicated that the difference between groups was statistically significant.

\section{Results}

TXET improves the learning and memory dysfunction of APPswe/PS1dE9 mice. APPswe/PS1dE9 mice exhibit cognitive damage and behavioral changes at 12 weeks old (25). The learning and memory abilities of mice were examined by the MWM. The results of the place navigation task are presented in Fig. 1. The average path length during the training days gradually declined. Compared with the control group, the path length and escape latency were significantly lengthened in the model group on day 5 ( $\mathrm{P}<0.05$; Fig. $1 \mathrm{~A}$ and $\mathrm{B})$. By contrast, the TXET high-dose, TXET low-dose and Aricept groups all exhibited significantly decreased escape latency and path length on day 5 compared with the model group (all $\mathrm{P}<0.05$ ). The results of the spatial probe test identified a significant reduction in the distance of the target quadrant between the model and control groups (Fig. $1 \mathrm{C} ; \mathrm{P}<0.05$ ). Compared with the model group, the TXET high-dose, TXET low-dose and Aricept groups significantly increased the distance of the target area (Fig. 1C; $\mathrm{P}<0.05$ ). These results indicate that TXET may improve learning and attenuate memory dysfunction.

TXET reduces the level and deposition of $A \beta$ in the hippocampi of APPswe/PS1dE9 mice. It has been demonstrated that APPswe/PS1dE9 mice exhibit plaque formation in the brain at the age of 17 weeks (25), making them a suitable model for 


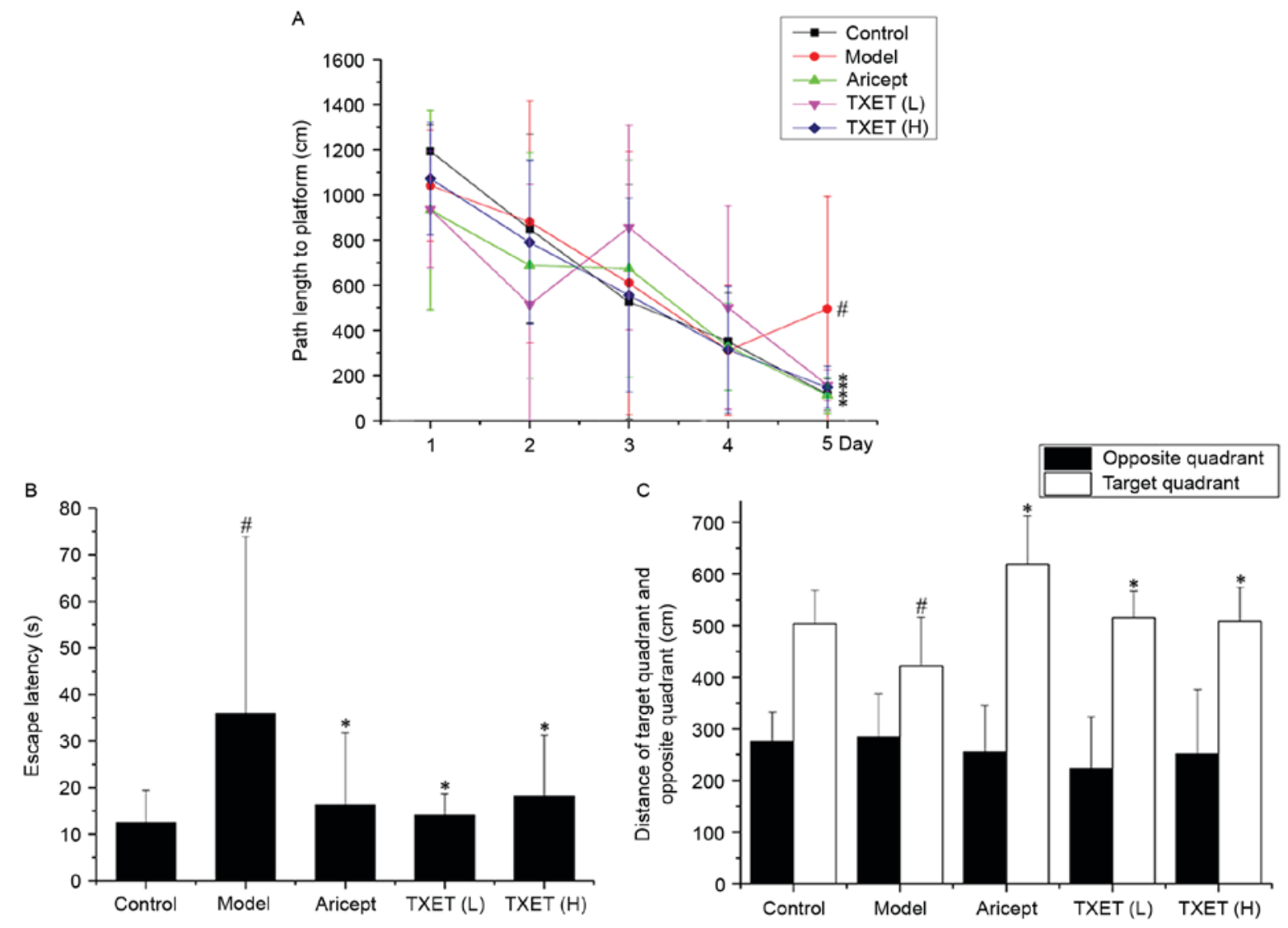

Figure 1. TXET ameliorates learning and memory dysfunction in APPswe/PS1dE9 mice. All mice were treated for 180 days and began the MWM on day 176 of treatment. (A) The path length during the 5 training days for the place navigation task. (B) The escape latency on day 5 of training for the place navigation task. (C) The distance of the target quadrant and opposite quadrant in the probe trail. $\mathrm{n}=8 /$ group. ${ }^{*} \mathrm{P}<0.05$ vs. the control group; ${ }^{*} \mathrm{P}<0.05$ vs. the model group. TXET, Tongluo Xingnao effervescent tablet; $\mathrm{H}$, high-dose; L, low-dose.

studying $\mathrm{A} \beta$ metabolism in AD. To evaluate the anti-AD effect of TXET in A $\beta$ metabolism, the effect of TXET on A $\beta$ levels and deposition was investigated. As presented in Fig. 2A, the number and size of the $A \beta$-positive plaques decreased in the hippocampi of AD mice following TXET administration. Statistical analysis revealed that low- and high-dose TXET $(\mathrm{P}<0.01)$ and Aricept $(\mathrm{P}<0.05)$ significantly reduced the number of $A \beta$-positive plaques compared with the model group (Fig. 2B). TXET treatment also reduced $A \beta$ levels. $A \beta$ levels in the hippocampus were measured by ELISA, which demonstrated that levels of $\mathrm{A} \beta_{1-40}$ were significantly lowered by $56.02 \%(\mathrm{P}<0.01)$ and $21.29 \%(\mathrm{P}<0.05)$ following treatment with high- and low-dose TXET, respectively, compared with the model group (Fig. $2 \mathrm{C}$ ). Levels of $\mathrm{A} \beta_{1-42}$ were also significantly reduced in the hippocampus by $66.14 \%(\mathrm{P}<0.01)$ and $21.62 \%(\mathrm{P}<0.05)$ following treatment with high- and low-dose TXET, respectively, compared with the model group (Fig. 2D). However, Aricept exhibited no significant effect on $A \beta$ concentration in the hippocampi of APPswe/PS1dE9 mice. These results suggest that TXET reduces $A \beta$ levels and deposition.

TXETreduces A $\beta$ by inhibiting $\beta$-secretase in APPswe/PSIdE9 mice. The cleavage pathway of APP serves a major role in A $\beta$ generation (9). The reduction of $\mathrm{A} \beta$ production may be associated with the regulation of APP processing; therefore, the effects of TXET on the expression of APP were examined.
It was demonstrated that the expression of APP was significantly decreased in mice administered with low- and high-dose TEXT, compared with the model group $(\mathrm{P}<0.01)$, indicating that TXET reduces $A \beta$ levels via the regulation of APP processing. APP is cleaved by $\alpha$ - or $\beta$-secretase via two cleavage pathways. Therefore, the protein levels of CTF- $\alpha$ and CTF- $\beta$ cleaved, respectively, by $\alpha$ - or $\beta$-secretase, were examined (Fig. 3). The data indicated that the expression of CTF- $\alpha$ significantly increased following treatment with Aricept compared with the model group $(\mathrm{P}<0.05)$, but no significant differences were identified following TXET administration. A significant reduction in the expression of CTF- $\beta$ was observed in the TXET-treated groups $(\mathrm{P}<0.01)$ compared with the model group. There were no significant differences between the expression of CTF- $\beta$ in the model and Aricept groups. These data suggest that TXET decreases $\mathrm{A} \beta$ production by regulating APP processing, which inhibits $\beta$-secretase.

The A $\beta$ reduction of TXET is irrelevant to the cleavage of $\gamma$-secretase in APPswe/PS1dE9 mice. The involvement of $\gamma$-secretase is the final step in APP processing and PS1 is a component of the $\gamma$-secretase complex (26). Thus, the effect of TXET treatment on the expression of PS1 and the activity of $\gamma$-secretase was examined (Fig. 4). The results demonstrated that TXET treatment did not significantly affect PS1 expression and $\gamma$-secretase activity. These data suggest that 
A

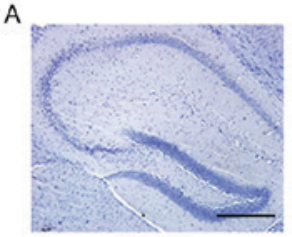

Control

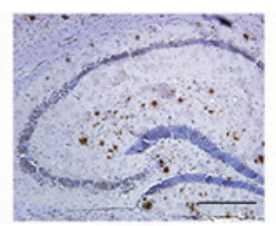

Model

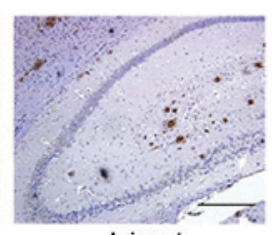

Aricept

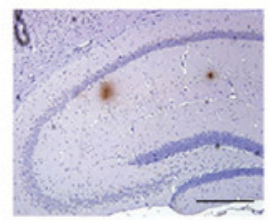

TXET (L)

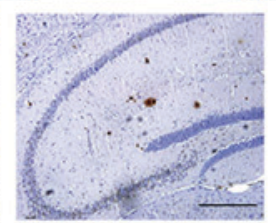

$\operatorname{TXET}(\mathrm{H})$

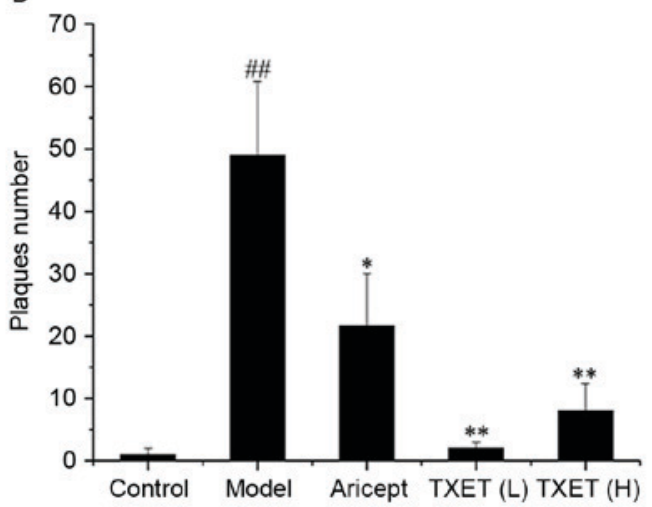

C

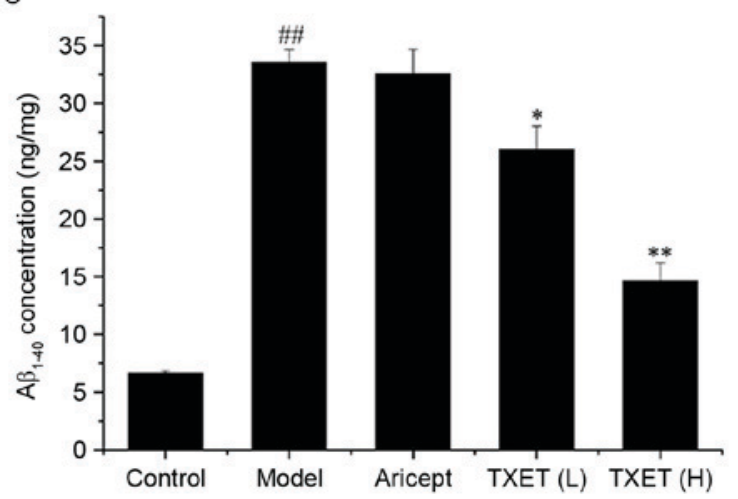

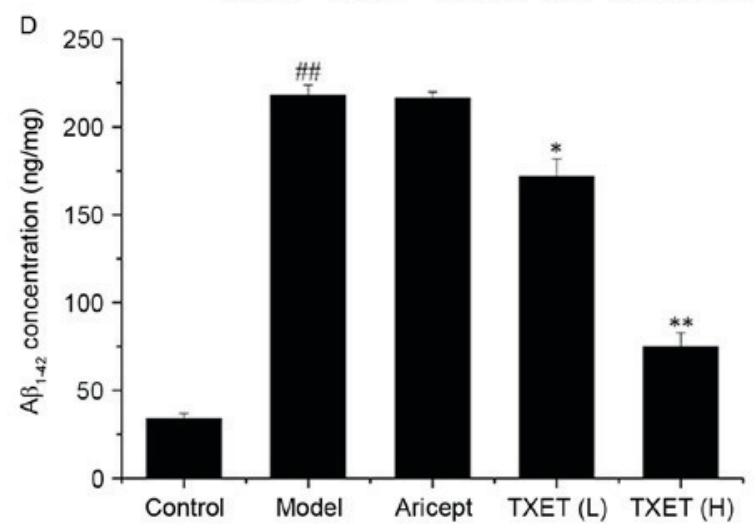

$0_{20}$

Figure 2. TXET reduces $A \beta$ deposition and levels of $A \beta_{1-40}$ and $A \beta_{1-42}$ in the brains of APPswe/PS1dE9 mice. (A) Immunohistochemical detection of $A \beta$ in the hippocampus ( $\mathrm{n}=3$ per group). A $\beta$-positive plaques were stained dark brown. Scale bars: $100 \mu \mathrm{m}$ (IHCx200). (B) Semi-quantitative analysis of the number of $A \beta$-positive plaques in the hippocampus. The content of (C) $A \beta_{1-40}$ and (D) $A \beta_{1-42}$ in the hippocampi of APPswe/PS1dE9 mice. $n=6 /$ group. ${ }^{\# \#} \mathrm{P}<0.01$ vs. the control group; " $\mathrm{P}<0.05,{ }^{* *} \mathrm{P}<0.01$ vs. the model group. $\mathrm{A} \beta$, Amyloid- $\beta$; TXET, Tongluo Xingnao effervescent tablet $\mathrm{H}$, high-dose; L, low-dose.

A

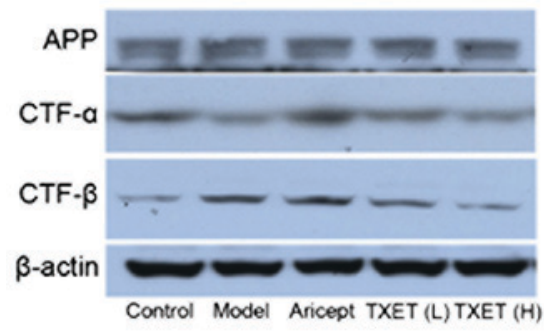

B

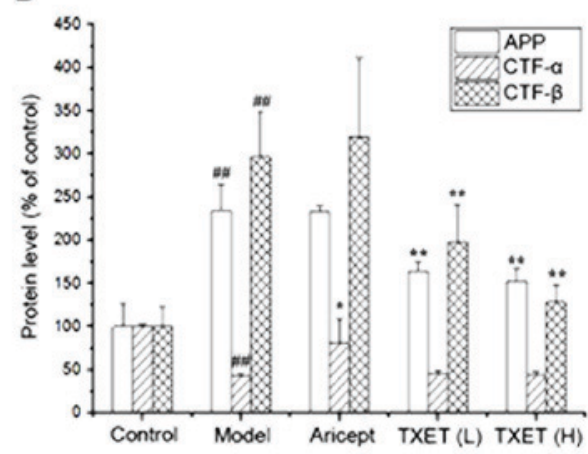

Figure 3. TXET regulates APP processing in APPswe/PS1dE9 mice. (A) Representative western blot analysis of APP, CTF- $\alpha$, CTF- $\beta$ and $\beta$-actin. (B) Densitometry analysis of the western blotting analysis. $\mathrm{n}=3$ /group. ${ }^{* \prime} \mathrm{P}<0.01$ vs. the control group; ${ }^{*} \mathrm{P}<0.05,{ }^{* *} \mathrm{P}<0.01$ vs. the model group. TXET, Tongluo Xingnao effervescent tablet; $\mathrm{H}$, high-dose; L, low-dose; APP, amyloid precursor protein; CTF, C-terminal fragment.

TXET does not reduce A $\beta$ levels in APPswe/PS1dE9 mice via the regulation of $\gamma$-secretase.

The reduced effect on A $\beta$ of TXET is not associated with NEP or IDE in APPswe/PSIdE9 mice. A $\beta$ levels in the brain are dependent on APP processing and A $\beta$ degradation. NEP and IDE are the most important $A \beta$-degrading enzymes $(12,13)$. TXET decreased $A \beta$ production by inhibiting $\beta$-secretase; therefore, the association between $\beta$-secretase and the $\mathrm{A} \beta$ degradation enzymes was investigated (Fig. 5). The results demonstrated that TXET treatment did not significantly affect the expression of NEP and IDE compared with the model group.

\section{Discussion}

The primary pathological change that occurs during AD is the excessive deposition of $A \beta$, caused by the decrease in metabolism that occurs during the aging process (27). A $\beta$ deposition is closely associated with $A \beta$ homeostasis (28). Under normal physiological conditions, the generation and elimination of $\mathrm{A} \beta$ maintains a dynamic balance. However, 

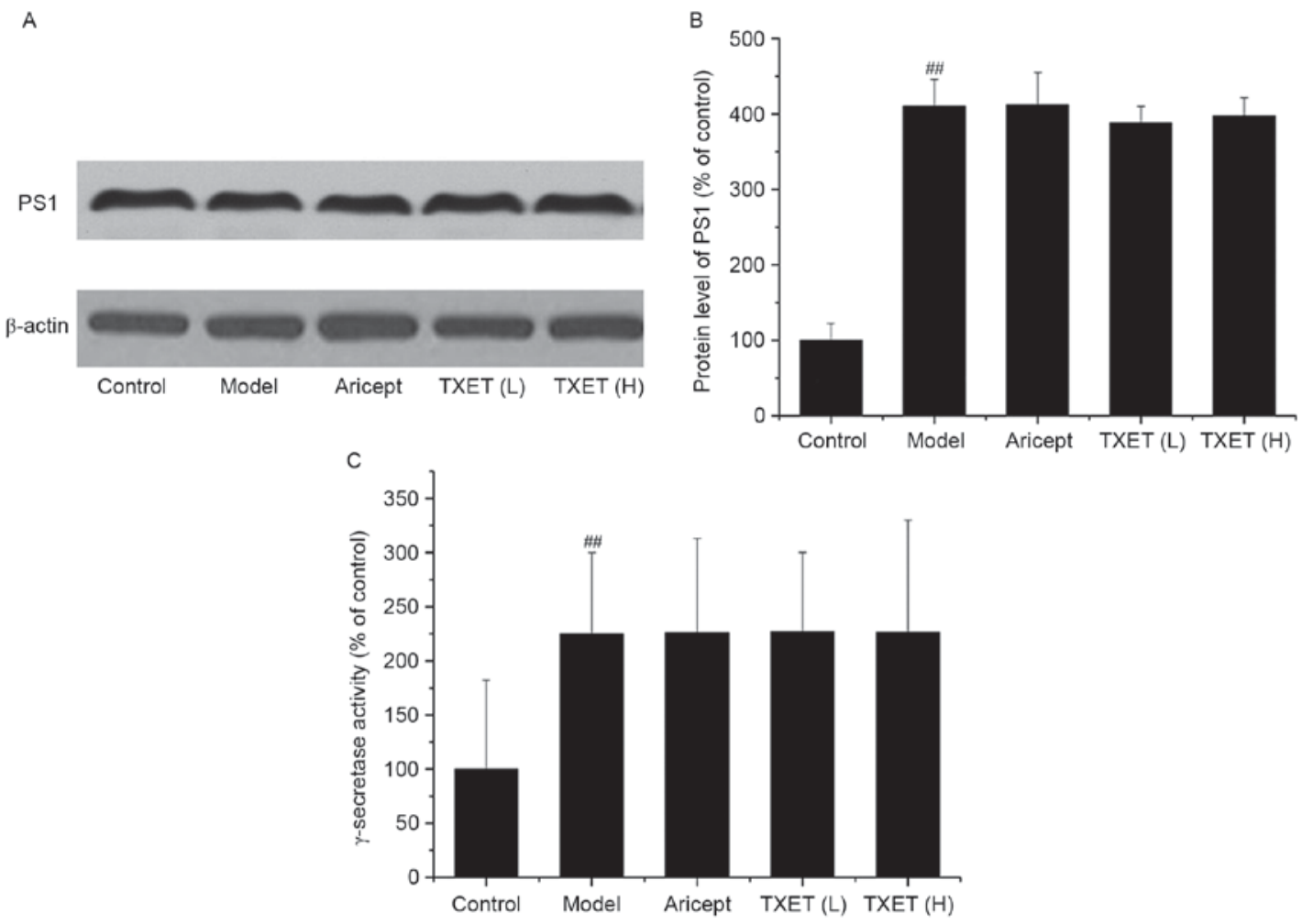

Figure 4. TXET does not regulate $\gamma$-secretase in APPswe/PS1dE9 mice. (A) Representative western blot analysis of PS1 and $\beta$-actin. (B) Densitometry analysis of the western blotting analysis. (C) Quantification of $\gamma$-secretase activity. $n=3 /$ group. ${ }^{\# \#} \mathrm{P}<0.01$ vs. the control group. TXET, Tongluo Xingnao effervescent tablet; H, high-dose; L, low-dose; PS1, presenilin-1.
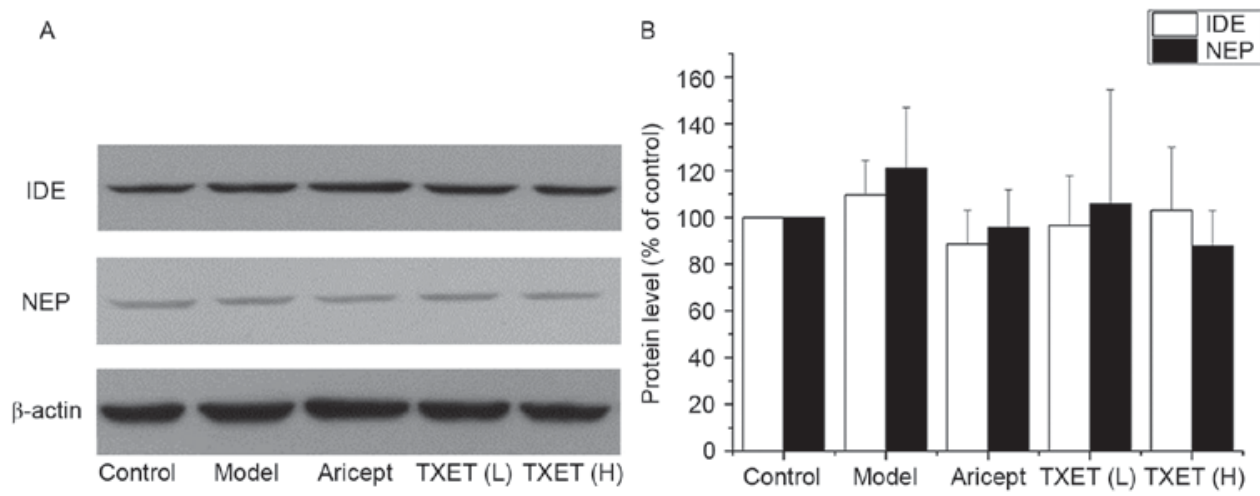

Figure 5. TXET does not regulate IDE or NEP in APPswe/PS1dE9 mice. (A) Representative western blot analysis of IDE, NEP and $\beta$-actin. (B) Densitometry analysis of the western blotting analysis. TXET, Tongluo Xingnao effervescent tablet; H, high-dose; L, low-dose; IDE, insulin-degrading enzyme; NEP, neprilysin.

if $\mathrm{A} \beta$ homeostasis is disrupted, $\mathrm{A} \beta$ may aggregate, resulting in the formation of neurofibrillary tangles, cell death and promoting the onset and development of AD. Therefore, decreasing $A \beta$ production and inhibiting $A \beta$ aggregation may be developed as novel therapeutic strategies to treat patients with AD.

Previous studies have indicated that reducing the formation of $\mathrm{A} \beta$ plaques in the brain is the key to $\mathrm{AD}$ therapy, thus verifying the $A \beta$ hypothesis of $A D(29,30)$. Reducing $A \beta$ levels depends on APP processing by $\beta$ - and $\gamma$-secretase, as well as $A \beta$ elimination via transport processes, cell-mediated clearance and $A \beta$ degradation (31). It has been reported that upregulating $\alpha$-secretase and downregulating $\beta$-secretase may decrease the generation of $A \beta$ (32). Furthermore, inhibition of $\gamma$-secretase may reduce $A \beta$ concentrations in the plasma and cerebrospinal fluid of patients with AD (33). Previous in vitro and in vivo studies have reported that altering catabolism may decrease $A \beta$ levels, including via NEP (34-36) and IDE $(37,38)$. However, there are currently no data regarding the long-term safety and efficacy of $A \beta$ inhibitors or associated drugs. Thus, it is important to identify reliable, effective and safe novel therapeutic strategies that may be used to treat patients with $\mathrm{AD}$.

Different TCM treatments have been widely used to treat various diseases and are safe and effective. It has been hypothesized that herbal medicine may improve cognitive 
dysfunction to treat AD (39-41). TCM defines dementia as a disease of abnormal consciousness, which is caused by mental dysfunction and deterioration of the brain tissue. TXET, based on the traditional Chinese formula Qionggui Tang plus Scutellaria baicalensis Georgi, is a patented prescription that has been used as a long-term treatment of patients with dementia at the Affiliated Hospital of Chengdu University of Traditional Chinese Medicine. It may be a reliable and effective method of treating AD (18-22,41-44).

The results of previous studies have revealed that TXET may improve dysfunction in learning and memory, improve cerebral metabolism and mitochondrial function (18), downregulate levels of CKD5 and GSK-3 to inhibit tau phosphorylation and promote hippocampal synaptophysin remodeling in AD rats $(19,42)$. Furthermore, TXET reduces $\mathrm{ACh}$ and $\mathrm{ChAT}$ to enhance the central cholinergic neuronal system in the AD rat hippocampus (20). It may also regulate the p38 and the mitogen-activated protein kinase signaling pathways in vascular dementia (VD), the second most common cause of dementia following AD (23). Additionally, it may increase levels of cytochrome $c$ oxidase in the hippocampus in VD (43) and in an AD model induced by chronic cerebral ischemia (44). Furthermore, TXET may increase the expression of IDE in the hippocampus of AD rats (20), suggesting that TXET may act to treat AD by altering the metabolism of $\mathrm{A} \beta$.

Transgenic APP695sw/PS1dE9 mice overexpressing APP695sw and PSEN1dE9 represent an ideal animal model to use to study the pathogenesis of $\mathrm{AD}$ and evaluate novel experimental therapeutics for AD (45). APP695sw/PS1dE9 mice overproduce $A \beta$ and exhibit cognitive damage and behavioral changes at 12 weeks of age, In addition, plaque formation occurs in their brains at the age of 17 weeks, which are beneficial for identifying the association between TXET and $\mathrm{A} \beta$ metabolism. A previous study indicated that TXET may be used to treat AD by altering the metabolism of $A \beta(20)$. The present study demonstrated that APP695sw/PS1dE9 mice in the model group exhibited learning and memory dysfunction, as well as the increased production of $\mathrm{A} \beta_{1-40}$, $\mathrm{A} \beta_{1-42}$ and plaques in the brain. However, TXET administration prolonged the escape latency, reduced the average path length in training days and the distance of the target area in mice undergoing MWM. Plaque formation, associated with $\mathrm{A} \beta$ metabolism, was inhibited following administration in the mice at 3-months-old (12 weeks) and TXET reduced the content of $A \beta_{1-40}, A \beta_{1-42}$ and the expression of plaques in the hippocampi of APP695sw/PS1dE9 mice. The result suggested that TXET may attenuate learning and memory dysfunction in $\mathrm{AD}$ by regulating $\mathrm{A} \beta$ metabolism in the brain.

Aricept is widely used to treat AD. It has been reported that Aricept may reverse attention deficits in the $3 \times$ Tg AD mouse model, which exhibits $A \beta$ plaques and neurofibrillary tangles in the brain (46). In the present study, it was indicated that Aricept improved learning and memory deficits, but had little effect on the content of $A \beta$ plaques or the expression of CTF- $\beta$, NEP and IDE. However, it was also demonstrated that Aricept reduced APP and increased CTF- $\alpha$ levels, indicating that Aricept may regulate APP processing by activating $\alpha$-secretase. Aricept, an AChE inhibitor, is known to increase cholinergic function in the cerebral cortex (47). However, the mechanism by which $\alpha$-secretase is regulated by Aricept requires further study.

It is hypothesized that $A \beta$ accumulation serves the most important role in the pathogenesis of AD; activating inflammatory factors, as well as promoting oxidative stress, tau phosphorylation and synaptic dysfunction (48). The results of the present study demonstrated that TXET may reduce the content of $A \beta_{1-40}, A \beta_{1-42}$ and the expression of plaques in the hippocampi of APP695sw/PS1dE9 mice. However, it remains unclear if $A \beta$ generation or elimination mediates the A $\beta$-reducing effect of TXET. The results of the current study indicate that TXET reduces the expression of APP, suggesting that TXET may decrease A $\beta$ levels via APP processing. The data also demonstrated that TXET reduces CTF- $\beta$ but has no effect on CTF- $\alpha$. This suggests that TXET may decrease A $\beta$ generation by inhibiting $\beta$-secretase but not $\alpha$-secretase. PS1 is a component of the $\gamma$-secretase complex, which is involved in the final step of $A \beta$ generation (26). The current study demonstrated that the expression of PS1 and the activity of $\gamma$-secretase were not significantly altered following TXET administration, suggesting that $\mathrm{A} \beta$ reduction does not regulate $\gamma$-secretase. Furthermore, there was no difference in the expression of NEP and IDE following TXET administration in APP695sw/PS1dE9 mice, suggesting that the A $\beta$-reducing effect of TXET is not associated with NEP and IDE. However, a previous study by our group indicated that TXET increases the expression of IDE in the hippocampus of AD rats (21), which is inconsistent with the results of the present study. The two models of AD used in the different studies were assessed and it was identified that the methods of analysis differed between them. This may have caused the results of these two studies to be inconsistent; however, further analysis is required for clarification. Overall, these results suggest that TXET may reduce $A \beta$ accumulation by downregulating $\beta$-secretase.

The results of the present study suggest that TXET may ameliorate cognitive dysfunction and decrease $A \beta$ levels, while regulating $A \beta$ metabolism by downregulating $\beta$-secretase. However, further studies are required to elucidate its underlying mechanism of action. The results have laid the foundation for the development of TCM with an inhibitor of $\beta$-secretase as a target.

\section{Acknowledgements}

The authors would like to thank Dr Wang Ping and Dr Fang Zeng for the assistance on manuscript revision. Thanks also to Ms Qian Wu, Ms Ting Pan, Mr Hang Zheng and Ms Lixia Qing for their technical support with experimental study and data collection.

\section{Funding}

The present study was supported by the National Natural Science Foundation of China (Key Program; grant no. 81430100), the Major National Science and Technology Projects Creation of Major New Drugs (grant no. 2013ZX09103002-008) and the Sichuan Province Outstanding Youth Academic Technology Leaders Subsidy Scheme (grant no. 2011JQ0014). 


\section{Availability of data and materials}

The analyzed datasets generated during the present study are available from the corresponding author on reasonable request.

\section{Authors' contributions}

SX provided experimental concepts and design, offered scientific direction and reviewed the manuscript. WF performed the research, the data collection and analysis and drafted the manuscript. TM and YD performed the research and aided manuscript revision. JW and HC performed the assessment of murine learning and memory abilities examined by MWM and participated in data collection. All authors participated in the manuscript preparation and read and approved the final manuscript.

\section{Ethics approval and consent to participate}

The treatment and care of animals adhered to the international laws and regulations indicated in the National Institutes of Health Guide for the Care and Use of Laboratory Animals. All animal protocols were approved by the Institutional Animal Care and Use Committee of Dongfang Hospital affiliated with the Beijing University of Chinese Medicine (Beijing, China).

\section{Consent for publication}

Not applicable.

\section{Competing interests}

All authors declare that they have no competing interests.

\section{References}

1. Wilhelmus MM, Otte-Höller I, Wesseling P, de Waal RM, Boelens WC and Verbeek MM: Specific association of small heat shock proteins with the pathological hallmarks of Alzheimer's disease brains. Neuropathol Appl Neurobiol 32: 119-130, 2006.

2. Reitz C, Brayne C and Mayeux R: Epidemiology of Alzheimer disease. Nat Rev Neurol 7: 137-152, 2011.

3. Golde TE: The Abeta hypothesis: Leading us to rationally-designed therapeutic strategies for the treatment or prevention of Alzheimer disease. Brain Pathol 15: 84-87, 2005.

4. Schenk D, Basi GS and Pangalos MN: Treatment strategies targeting amyloid $\beta$-protein. Cold Spring Harb Perspect Med 2: a006387, 2012

5. Selkoe DJ and Hardy J: The amyloid hypothesis of Alzheimer's disease at 25 years. EMBO Mol Med 8: 595-608, 2016.

6. Lane RF, Shineman DW, Steele JW, Lee LB and Fillit HM: Beyond amyloid: The future of therapeutics for Alzheimer's disease. Adv Pharmacol 64: 213-271, 2012.

7. Obregon D, Hou H, Deng J, Giunta B, Tian J, Darlington D, Shahaduzzaman M, Zhu Y, Mori T, Mattson MP and Tan J: Soluble amyloid precursor protein- $\alpha$ modulates $\beta$-secretase activity and amyloid- $\beta$ generation. Nat Commun 3: $777,2012$.

8. George-Hyslop S and Fraser PE: Assembly of the presenilin $\gamma$-/ع-secretase complex. J Neurochem 120 (Suppl 1): S84-S88, 2012.

9. Zhang HM, Zhang X and Li Y: The regulation of curcumin on the amyloidogentic pathaway of APP in Alzheimer's disease. Chin Pharmacol Bull 25: 361-366, 2009 (In Chinese).
10. Grimm MO, Mett J, Stahlmann CP, Haupenthal VJ, Zimmer VC and Hartmann T: Neprilysin and $A \beta$ clearance: Impact of the APP intracellular domain in NEP regulation and implications in Alzheimer's disease. Front Aging Neurosci 5: 98, 2013.

11. Wang DS, Dickson DW and Malter JS: Beta-Amyloid degradation and Alzheimer's disease. J Biomed Biotechnol 2006: 58406, 2006.

12. Kanemitsu H, Tomiyama T and Mori H: Human neprilysin is capable of degrading amyloid beta peptide not only in the monomeric form but also the pathological oligomeric form. Neurosci Lett 350: 113-116, 2003.

13. Kurochkin IV and Goto S: Alzheimer's beta-amyloid peptide specifically interacts with and is degraded by insulin degrading enzyme. FEBS Lett 345: 33-37, 1994.

14. Hayes CD, Dey D, Palavicini JP, Wang H, Patkar KA, Minond D, Nefzi A and Lakshmana MK: Striking reduction of amyloid plaque burden in an Alzheimer's mouse model after chronic administration of carmustine. BMC Med 11: 81, 2013.

15. Eckman EA and Eckman CB: Abeta-degrading enzymes: Modulators of Alzheimer's disease pathogenesis and targets for therapeutic intervention. Biochem Soc Trans 33: 1101-1105, 2005.

16. Siemers ER, Sundell KL, Carlson C, Case M, Sethuraman G, Liu-Seifert H, Dowsett SA, Pontecorvo MJ, Dean RA and Demattos R: Phase 3 solanezumab trials: Secondary outcomes in mild Alzheimer's disease patients. Alzheimers Dement 12: 110-120, 2016.

17. Turtle CJ, Hanafi LA, Berger C, Hudecek M, Pender B, Robinson E, Hawkins R, Chaney C, Cherian S, Chen X, et al: Immunotherapy of non-Hodgkin's lymphoma with a defined ratio of $\mathrm{CD} 8+$ and $\mathrm{CD} 4+\mathrm{CD} 19$-specific chimeric antigen receptor-modified T cells. Sci Transl Med 8: 355ra116, 2016.

18. Dai Y, Ma T, Ren X, Wei J, Fu W, Ma Y, Xu S and Zhang Z: Tongluo Xingnao Effervescent Tablet preserves mitochondrial energy metabolism and attenuates cognition deficits in APPswe/PS1De9 mice. Neurosci Lett 630: 101-108, 2016.

19. Xu SJ, Dai Y, Zhang YJ, Xiong M, Ma YT and Zhong ZD: Effects of tongluo xingnao effervescent tablet on expression of CKD5 and GSK-3 in rat model of hippocampus with $A \beta$ injection. West China J Pharm Sci 28: 140-140, 2013 (In Chinese).

20. Zhang YJ, Ju SH, Hu Y, Ren XY and Xu SJ: Effect of Tongluo Xingnao effervescent tablets on cerebral cholinergic function of mice dementia model induced by scopolamine. Tradit Chin Drug Res Pharmacol 25: 272-276, 2014 (In Chinese).

21. Zhang YJ, Dai Y, Hu Y, Ma YT, Xu SJ, and Wang YY: Effect of tongluo xingnao effervescent tablet on learning and memory of AD rats and expression of insulin-degrading enzyme in hippocampus. Zhongguo Zhong Yao Za Zhi 38: 2863-2867, 2013 (In Chinese).

22. Xu SJ and Dai Y: A pharmaceutical composition for treating neurodegenerative diseases and its preparation method and application. CN Patent CN103181954A. Filed April 12, 2013; issued July 3, 2013 (In Chinese).

23. Ren X, Wei J, Gong D, Hu Y, Chen $\mathrm{H}$ and Xu S: Tongluoxingnao effervescent tablets ameliorate learning and memory impairment in a rat model of vascular dementia via the regulation of the p38 and ERK MAPK signaling pathways. Int J Clin Exp Med 9: 5400-5412, 2016.

24. Institute of Laboratory Animal Resources (US), Committee on Care and Use of Laboratory Animals, National Institutes of Health (US), and Division of Research Resources: Guide for the care and use of laboratory animals. The National Academies Press, Washington, DC, 1985.

25. Buttini M, Masliah E, Barbour R, Grajeda H, Motter R, Johnson-Wood K, Khan K, Seubert P, Freedman S, Schenk D and Games D: Beta-amyloid immunotherapy prevents synaptic degeneration in a mouse model of Alzheimer's disease. J Neurosci 25: 9096-9101, 2005

26. Li T, Li YM, Ahn K, Price DL, Sisodia SS and Wong PC: Increased expression of PS1 is sufficient to elevate the level and activity of $\gamma$-secretase in vivo. PLoS One 6: e28179, 2011.

27. Grimm A, Friedland K and Eckert A: Mitochondrial dysfunction: The missing link between aging and sporadic Alzheimer's disease. Biogerontology 17: 281-296, 2016.

28. Selkoe DJ and Hardy J: The amyloid hypothesis of Alzheimer's disease at 25 years. EMBO Mol Med 8: 595-608, 2016.

29. Izzo NJ, Staniszewski A, To L, Fa M, Teich AF, Saeed F, Wostein H, Walko T III, Vaswani A, Wardius M, et al: Alzheimer's therapeutics targeting Amyloid beta 1-42 oligomers I: Abeta 42 oligomer binding to specific neuronal receptors is displaced by drug candidates that improve cognitive deficits. PLoS One 9: e111898, 2014. 
30. Sevigny J, Chiao P, Bussière T, Weinreb PH, Williams L, Maier M, Dunstan R, Salloway S, Chen T, Ling Y, et al: The antibody aducanumab reduces $\mathrm{A} \beta$ plaques in Alzheimer's disease. Nature 537: 50-56, 2016.

31. Wang YJ, Zhou HD and Zhou X F: Clearance of amyloid-beta in Alzheimer's disease: Progress, problems and perspectives. Drug Discov Today 11: 931-938, 2006.

32. Liu XP, Qian X, Xie Y, Qi Y, Peng MF, Zhan BC and Lou ZQ: Betaine suppressed $A \beta$ generation by altering amyloid precursor protein processing. Neurol Sci 35: 1009-1013, 2014.

33. Siemers ER, Quinn JF, Kaye J, Farlow MR, Porsteinsson A, Tariot P, Zoulnouni P, Galvin JE, Holtzman DM, Knopman DS, et al: Effects of a gamma-secretase inhibitor in a randomized study of patients with Alzheimer disease. Neurology 66: 602-604, 2006.

34. Iwata N, Tsubuki S, Takaki Y, Watanabe K, Sekiguchi M, Hosoki E, Kawashima-Morishima M, Lee HJ, Hama E, Sekine-Aizawa Y and Saido TC: Identification of the major Abeta1-42-degrading catabolic pathway in brain parenchyma: Suppression leads to biochemical and pathological deposition. Nat Med 6: 143-150, 2000.

35. El-Amouri SS, Zhu H, Yu J, Gage FH, Verma IM and Kindy MS Neprilysin protects neurons against Abeta peptide toxicity. Brain Res 1152: 191-200, 2007.

36. Marr RA, Rockenstein E, Mukherjee A, Kindy MS, Hersh LB, Gage FH, Verma IM and Masliah E: Neprilysin gene transfer reduces human amyloid pathology in transgenic mice. J Neurosci 23: 1992-1996, 2003.

37. Bulloj A, Leal MC, Surace EI, Zhang X, Xu H, Ledesma MD, Castaño EM and Morelli L: Detergent resistant membrane-associated IDE in brain tissue and cultured cells: Relevance to Abeta and insulin degradation. Mol Neurodegener 3: 22, 2008.

38. Farris W, Mansourian S, Chang Y, Lindsley L, Eckman EA, Frosch MP, Eckman CB, Tanzi RE, Selkoe DJ and Guenette S: Insulin-degrading enzyme regulates the levels of insulin, amyloid beta-protein, and the beta-amyloid precursor protein intracellular domain in vivo. Proc Natl Acad Sci USA 100 4162-4167, 2003.
39. Wu TY, Chen CP and Jinn TR: Traditional Chinese medicines and Alzheimer's disease. Taiwan J Obstet Gynecol 50: 131-135, 2011.

40. Howes MJR and Houghton PJ: Plants used in Chinese and Indian traditional medicine for improvement of memory and cognitive function. Pharmacol Biochem Behav 75: 513-527, 2003.

41. Dos Santos-Neto LL, de Vilhena Toledo MA, Medeiros-Souza P and de Souza GA: The use of herbal medicine in Alzheimer's disease: a systematic review. Evid Based Complement Alternat Med 3: 441-445, 2006.

42. Zhang YJ, Xu SJ, Dai Y, Xiong M and Ma YT: Effects of TongluoxingnaoEffervescent Tablet on synaptophysin of injecting $\mathrm{A} \beta$ in the hippocampus rat model. Pharm Clin Chin Mat Med 28: 84-87,2012 (In Chinese).

43. Ren XY, Hu Y, Wei JP, Ma YT and Xu SJ: Effects of Tongluo Xingnao effervescent tablets on expression of cytochrome $\mathrm{C}$ oxidase in cells in hippocampus of VD rat model. Chin J Basic Med Tradit Chin Med 2: 159-161, 2015 (In Chinese).

44. Ren XY, Hu Y, Wei JP, Fu WJ, Xu SJ and Wang YY: Effects of Tongluo Xingnao effervescent tablets on blood rheology, iNOS, VEGF and LDH-5 in MID rats. Zhongguo Zhong Yao Za Zhi 41: 1119-1123, 2016 (In Chinese).

45. Huang $\mathrm{H}$, Nie S, Cao M, Marshall C, Gao J, Xiao N, $\mathrm{Hu} \mathrm{G}$ and Xiao M: Characterization of AD-like phenotype in aged APPSwe/PS1dE9 mice. Age (Dordr) 38: 303-322, 2016.

46. Romberg C, Mattson MP, Mughal MR, Bussey TJ and Saksida LM: Impaired attention in the 3xTgAD mouse model of Alzheimer's disease: Rescue by donepezil (Aricept). J Neurosci 31: 3500-3507, 2011.

47. Stahl SM: The new cholinesterase inhibitors for Alzheimer's disease, Part 1: Their similarities are different. J Clin Psychiatry 61: 710-711, 2000.

48. Gandy S: The role of cerebral amyloid beta accumulation in common forms of Alzheimer disease. J Clin Invest 115: 1121-1129, 2005. 